Introduction Flow-diverter stents (FDSs) have been used effectively to treat wide neck and complex intracranial aneurysms (IAs). However, treatment strategies vary significantly. Previously, the normalized mean aneurysm flow amplitude ratio (MAFA-R) has been described as a novel metric to quantify aneurysm flow reduction that occurs as a result of FD stenting. The aim of this study was to evaluate the performance of the MAFA-R in predicting complete occlusion for IAs treated with FDSs.

Materials and methods We included patients harboring unruptured saccular IAs, which were treated with flow diverter stents. For each patient, a 3D rotational angiogram was performed prior to implantation and two high frame rate DSA sequences were acquired respectively pre- and post- FD stenting. The aneurysmal flow reduction as indicated by the MAFA-R was calculated using dedicated software (AneurysmFlow, Philips Healthcare). The MAFA-R performance for prediction of complete aneurysm occlusion was tested against the thrombosis incidence at 3, 6, and 12 months by using the receiver operating characteristic (ROC) analysis performance test. The AUC was determined, as well as the threshold maximizing the sum of sensitivity and specificity. The analysis was carried out on the total aneurysm cohort as well as for a subgroup of aneurysms $>10 \mathrm{~mm}$.

Results We included 54 consecutive patients. All cases were successfully treated with a[o1] flow diverter stent. At 3 months follow-up, 11 out of 54 aneurysms were fully occluded (20\%), and 43 displayed residual circulation (80\%). The performance of MAFA-R test for the total cohort was: AUC $=0.60, p=0.386$ and threshold $=0.62$. At 6 months $31 / 54$ aneurysms occluded (57\%), AUC $=0.657, \mathrm{p}=0.414$, threshold $=0.93$. At 12 months $39 / 52$ aneurysms occluded (75\%), $\mathrm{AUC}=0.66, \mathrm{p}=0.081$, threshold $=0.89$. For the large aneurysm subgroup, the results were: at 3 months 2/19 aneurysms occluded (11\%): AUC $=0.97, \mathrm{p}=0.014$, threshold $=0.64$; at 6 months $7 / 19$ aneurysms occluded (37\%): AUC $=0.82, \mathrm{p}<0.0001$, threshold $=0.93$; and at 12 months 11/18 aneurysms occluded (61\%): AUC $=0.91$, $\mathrm{p}<0.0001$, threshold $=0.89$.

Conclusions Aneurysm occlusion after FD stenting is determined by multiple factors including aneurysm size, morphology and the hemodynamic environment. While for the total aneurysm cohort no significance was reached, the results of this study show that for large aneurysms, the MAFA-R is an independent predictor of aneurysm occlusion at 12 months follow up (MAFA-R threshold $=0.89$ ). This real-time functional assessment may potentially help the clinician to adapt the treatment during the procedure by adding further FDS layers or intra-saccular coils. [o1]Some cases were implanted with 2 devices, BB4-4AE2-9764-89B1B81991A1\$\$

Disclosures V. Mendes Pereira: 1; C; Philips, Medtronic. O. Brina: None. J. Bracken: 5; C; Philips. T. Krings: None. K. Lovblad: None. F. van Nijnatten: 5; C; Philips. D. Ruijters: 5; C; Philips. T. Grünhagen: 5; C; Philips.

\section{E-082 VENOUS VASCULAR MALFORMATION OF THE FLOOR OF MOUTH MASQUERADING AS DERMOID TUMOR}

${ }^{1} \mathrm{~A}$ Dmytriw, ${ }^{2}$ S Song, ${ }^{3} \mathrm{P}$ Gullane, ${ }^{4} \mathrm{~K}$ terBrugge, ${ }^{5} \mathrm{E}$ Yu. ${ }^{1}$ Department of Medical Imaging, University of Toronto, Toronto, ON, Canada; ${ }^{2}$ Department of Otolaryngology, University of Toronto, Toronto, ON, Canada; ${ }^{3}$ Department of Otolaryngology, Princess Margaret Hospital, Toronto, ON, Canada; ${ }^{4}$ Department of Medical Imaging, Toronto Western Hospital, Toronto, ON, Canada; ${ }^{5}$ Department of Medical Imaging, Princess Margaret Hospital, Toronto, ON, Canada

\subsection{6/neurintsurg-2016-012589.154}

Introduction Venous vascular malformations (VVMs) are described as abnormal post-capillary lesions which exhibit low flow. These are typically malleable and may grow with endocrine fluctuations. These irregular conduits are often characterized by a soft, compressible, nonpulsatile tissue mass that may exhibit a blue tinge due to the collection of ectatic venous channels underneath the dermis. Further findings upon physical examination may include expansion of the malformation with the Valsalva maneuver, and flattening of the protrusion with applied pressure. A VVM that mimics the classic appearance of dermoid tumor on imaging has never been reported.

Case presentation We encountered a 43 year-old woman with intermittent dysphagia relating to a firm submandibular mass. Physical exam and cross-sectional imaging revealed features consistent with variant dermoid cyst. Gadolinium-enhanced MRI of the mass in T1 and T2 weighted imaging (Figure 1) revealed a lesion with T1 hypersensitivity and variable T2 signal. These results were followed-up with CT angiography which showed internal enhancement (Figure 1) possibly attributable to saponification, calcification, or rupture of the presumed dermoid cyst. However this pattern of enhancement was felt to be unusual, and digital subtraction angiography was performed to exclude a primarily vascular lesion. Catheter angiography eventually demonstrated a VVM which possessed vessels of variable size and partial thrombosis.

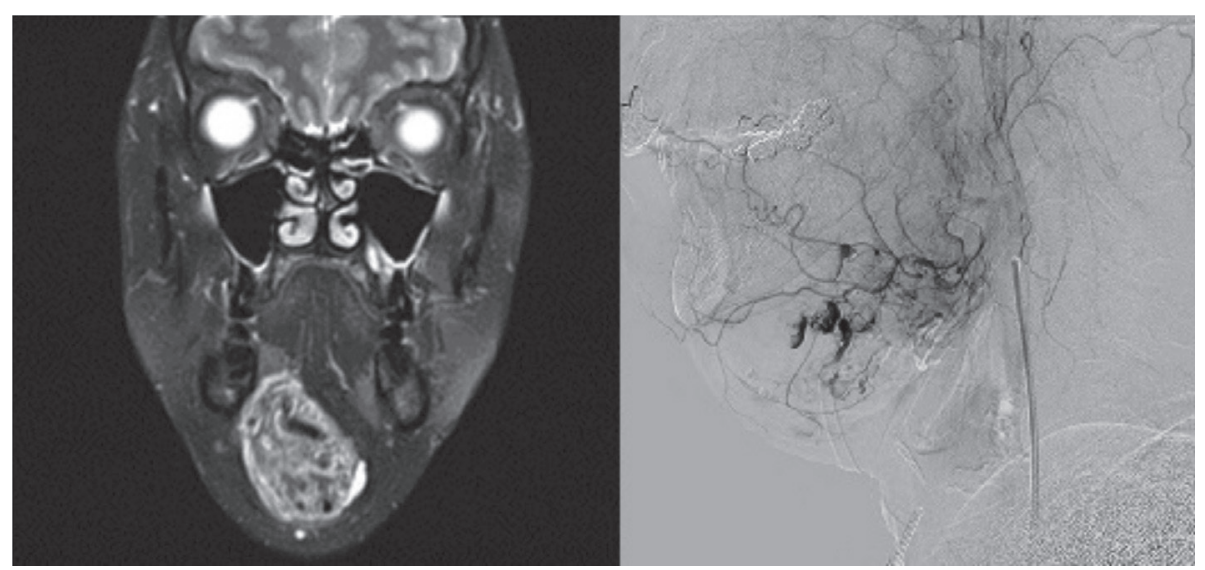

Abstract E-082 Figure 1 
Discussion This case presented a significant diagnostic challenge to our team as the VVM possessed numerous idiosyncratic features prevalent in cases of dermoid cysts, with simultaneous findings that are contraindicative of most venous malformations. The regression of the VVM during the patient's pregnancy was counterintuitive to expectation, as endocrine fluctuations would be expected to precipitate rapid enlargement of the lesion. Similarly, the abnormal firmness of the mass discouraged the diagnosis of a VVM which is characteristically soft on palpation, but this may be explained by the presence of partially thrombosed vessels. We report the case and propose that catheter angiography remains important in cases where vascular malformation is considered.

Disclosures A. Dmytriw: None. J. Song: None. P. Gullane: None. K. terBrugge: None. E. Yu: None.

\section{E-083 ACUTE STROKE INTERVENTION FOR LARGE VESSEL OCCLUSION WITH COMBINED STENT RETRIEVER AND SUCTION THROMBECTOMY (SOLUMBRA TECHNIQUE): A RETROSPECTIVE ANALYSIS OF 85 PATIENTS}

${ }^{1} \mathrm{~J}$ Wong, ${ }^{2} \mathrm{~N}$ Telischak, ${ }^{2} \mathrm{~J}$ Heit, ${ }^{1} \mathrm{~A}$ Moraff, ${ }^{1} \mathrm{R}$ Dodd, ${ }^{2} \mathrm{H}$ Do, ${ }^{2} \mathrm{M}$ Marks. ${ }^{1}$ Neurosurgery, Stanford, Stanford, $C A ;{ }^{2}$ Radiology, Stanford, Stanford, CA

\subsection{6/neurintsurg-2016-012589.155}

Introduction The superiority of mechanical thrombectomy over medical management has been established in recent randomized controlled trials, however, controversy remains over the most effective and safest technique for clot retrieval. Mechanical thrombectomy for large vessel occlusion (LVO) can be performed through aspiration alone with the ADAPT technique, or combined stent retriever with aspiration either in the cervical vasculature using a proximal balloon guide, or directly at the clot face with an intermediate catheter, the so-called "Solumbra" technique. The purpose of this study was to evaluate the efficacy and safety of the Solumbra technique at our institution.

Methods A retrospective chart review was conducted of all patients undergoing endovascular stroke treatment for LVO using the Solumbra technique between January 2014 and March 2016. The Solumbra technique consisted of deployment of a stent retriever (Solitaire, Trevo or Mindframe) distal to the clot with an intermediate catheter (5 Max Ace, Ace 64 or SOFIA) at the clot face. Patient demographic data, stroke presentation, treatment details, and complications were recorded. The primary outcome was successful TICI 2 B/3 reperfusion and the number of passes for revascularization. Secondary outcome measures were complication rates, including symptomatic intracranial hemorrhage ( $\mathrm{PH} 2$ hemorrhage with a NIHSS increase $>4$ ) and mRS $\leq 2$ on discharge. Embolization into new territory was based on new post-procedure MRI DWI lesions.

Results The Solumbra technique was performed for LVO in 85 patients (37 male, 48 female) with a mean age of 71.4 years (range 28-93). The mean NIHSS on presentation was 14 (IQR 10-19), and IV tPA was administered in 65\% of patients. Vessel occlusion was located predominantly in the anterior circulation in the ICA terminus ( $\mathrm{n}=12,14 \%)$, M1 ( $\mathrm{n}=51,60 \%)$ and M2 ( $\mathrm{n}=16,19 \%)$ segments, with Basilar occlusion occurring in 7 patients (8\%). Successful reperfusion (TICI $2 \mathrm{~B} / 3$ ) was achieved in $88 \%$ of patients, consisting of TICI 3 in 38 patients (45\%). The mean number of passes for revascularization was 1.9 (SD 1.3) and time from access to revascularization was 52.7 minutes (95\% CI 45.3-60.1). Mean NIHSS on discharge was 7 (IQR 2-12) with good functional outcome $(\mathrm{mRS} \leq 2)$ present in $44 \%$ of patients, and mortality $(\mathrm{mRS}=6)$ rate of $12 \%$. Complications included parenchymal hemorrhagic transformation (18\%), symptomatic intracranial hemorrhage $(4.7 \%)$ and emboli in new territory based on new MRI lesions (7\%).

Conclusion The Solumbra technique compares favourably to other thrombectomy techniques used for acute stroke intervention. It achieved a TICI $2 \mathrm{~B} / 3$ reperfusion in $88 \%$ of patients, with an average number of 1.9 passes. Good functional outcome was achieved in $44 \%$ of patients on discharge, with a symptomatic intracranial hemorrhage rate of $4.7 \%$.

Disclosures J. Wong: None. N. Telischak: None. J. Heit: None. A. Moraff: None. R. Dodd: None. H. Do: None. M. Marks: None.

\section{E-084 LONG-TERM CLINICAL OUTCOMES IN PATIENTS WITH ACUTE ISCHEMIC STROKES TREATED WITH MECHANICAL THROMBECTOMY WHO HAD MODERATE OR MODERATE SEVERE DISABILITY AT 90 DAYS}

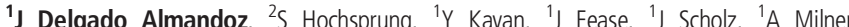
${ }^{2} \mathrm{P}$ Roohani, ${ }^{3} \mathrm{M}$ Mulder, ${ }^{2} \mathrm{~T}$ Hehr, ${ }^{2} \mathrm{R}$ Tarrel, ${ }^{4} \mathrm{D}$ Chappuis, ${ }^{2} \mathrm{M}$ Young. ${ }^{1}$ Neurointerventional Radiology, Abbott Northwestern Hospital, Minneapolis, MN; ${ }^{2}$ Vascular Neurology, Abbott Northwestern Hospital, Minneapolis, MN; ${ }^{3}$ Critical Care Medicine, Abbott Northwestern Hospital, Minneapolis, MN; ${ }^{4}$ Courage Kenny Rehabilitation Institute, Abbott Northwestern Hospital, Minneapolis, MN

\subsection{6/neurintsurg-2016-012589.156}

Background and purpose Long-term clinical outcomes in patients with acute ischemic stroke (AIS) treated with mechanical thrombectomy (MT) are not well understood. This study aims to determine long-term clinical outcomes in a consecutive cohort of patients with AIS treated with MT who had moderate or moderate severe disability at 90 day follow-up.

Methods Our prospectively-maintained institutional neurointerventional database was queried to identify all patients with AIS treated with MT at our institution from July 1st, 2011 until September 30th, 2015 who had moderate (modified Rankin Scale, mRS, 3) or moderate severe (mRS 4) disability at the time of the 90 day follow-up. At least 6 months after stroke onset, medical records were reviewed and/or phone interviews were conducted by a nurse certified in the mRS and independent of the MT procedure to determine the mRS at the time of last clinical contact. Baseline clinical and radiological characteristics were also obtained.

Results Forty patients met the study's inclusion criteria, comprising 29\% of patients treated with MT at our institution during the study period. Twenty patients were male $(50 \%)$ and 20 female $(50 \%)$. At presentation, 14 patients had a history of atrial fibrillation (35\%), 28 hypertension (70\%) and 13 diabetes mellitus (33\%). Mean age was 69.8 years. Mean admission NIHSS was 18.2. Twenty-five patients received intravenous tissue plasminogen activator (63\%). Thrombus location was M1 segment in 18 patients (45\%), internal carotid artery terminus in 14 patients $(35 \%)$, basilar artery in 4 patients (10\%) and M2/M3 segment in 4 patients (10\%). Discharge disposition was acute inpatient rehabilitation in 26 patients $(65 \%)$ and skilled nursing facility in 14 patients (35\%). At the time of the 90 day follow-up visit, 18 patients had an mRS of $3(45 \%)$ and 22 had an mRS of $4(55 \%)$. All 